EPiC Series in Engineering
Volume 3, 2018, Pages 731-738
HIC 2018. 13th International
Conference on Hydroinformatics

\title{
Research and development of flood control operation system of Beijing city, China
}

\author{
Chai Fuxin ${ }^{1,2}{ }^{*}$, Liu Shu ${ }^{1}$, Hu Changwei ${ }^{1}$ and Zhang Hongping ${ }^{1}$ \\ ${ }^{1}$ China institute of water resources and hydropower research \\ ${ }^{2}$ State Key Laboratory of Simulation and Regulation of Water Cycle in River Basin, PR China \\ chaifx@iwhr.com, fcds2001@iwhr.com, china_huchangwei@tom.com \\ luckyzhanghp@iwhr.com
}

\begin{abstract}
Assembling all the data of weather, rainfall, hydrology, engineering, water logging and other real-time information and basic information of the Beijing, the flood forecasting coupling model with hydrological and hydrodynamic method and the operation model of gate and dam are constructed. Obeying object-oriented open design concept, Beijing flood control operation system based on $\mathrm{B} / \mathrm{S}$ framework is developed, using Java language and database technology, which has achieved these functions that the analysis of flood control situation, flood forecast, flood control operation, flood simulation, evaluation of regulation results and scheduling management etc. At the same time, the mobile application client is developed by using html5 technology, with the functions of flood information query, mobile patrol and dangerous case report etc. All these can provide the decision-making basis for the integrated flood control management of Beijing city.
\end{abstract}

\section{Introduction}

Because of climate warming and La Niña effect in the world, the extreme rainfall in Beijing is increasing. At the same time, the comprehensive impact of increasing of impermeable area from the high degree of urbanization, speeded-up of rainfall production and convergence, the local rainstorm and floods happen frequently in Beijing, all these have result very big social and economic losses. From 1991 to 2016, there were 7 serious floods and 13 times more serious urban waterlogging in Beijing. Especially on July 21, 2012, the rainstorm and flood occurred in the southwest of Beijing has caused great losses for socio-economic and people's lives and property. Research on these fields that the flood control operation system of reservoir (Ref.1 to Ref.3) and flood control decision supporting system (Ref.4 to Ref.5) are more, and there is few research on flood control operation system for urban flooding. Aiming on the impact of the increasing rainstorm and flood disaster in Beijing, the

${ }^{*}$ Chai Fuxin, chaifx@iwhr.com 
basin as the object, the key projects being stressed, and upstream and downstream being overall considered, the rainstorm and flood control operation system is founded, which can effectively alleviate the water safety problems caused by local rainstorm, and can realize the fine management of flood control operation. All these have very important significance. Starting with data collection, model construction and system development, the basin being as the object, the key projects being stressed, and upstream and downstream, external flood and waterlogging being overall considered, the basin flood control operation system is founded, which can help to build a scientific flood control command system in Beijing.

\section{Models and methods}

The basin flood control operation models achieves multi-model coupling that conclude rainfallrunoff model, surface convergence model, river convergence model, reservoir operation model, model for sluice and rubber dam, and managing model for detention basin. The overall structure of the models is shown by Error! Reference source not found..

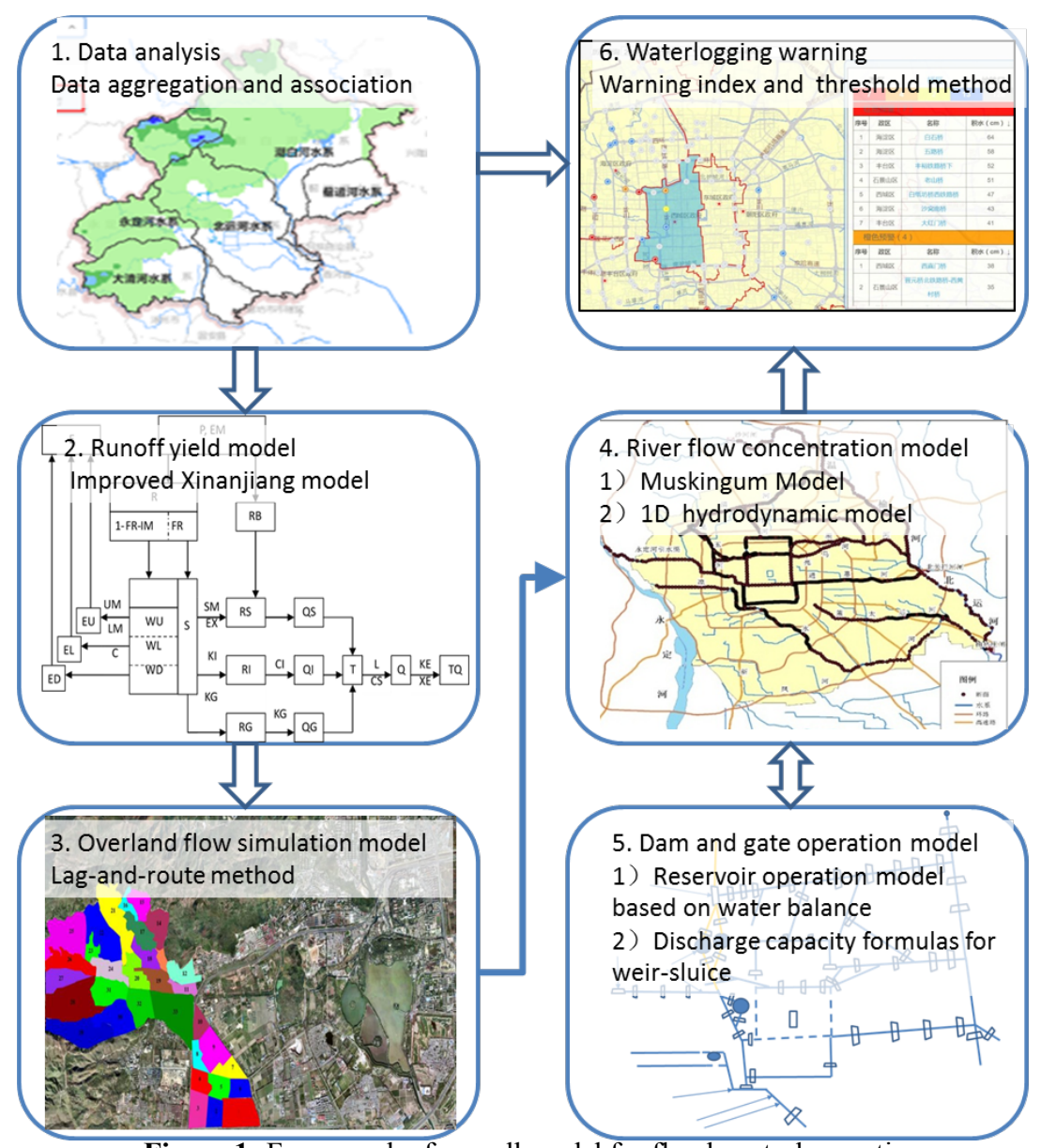

Figure 1: Framework of overall model for flood control operation 


\subsection{Runoff yield model}

Based on the model of Xin'anjiang, using a vertical mixed runoff producing pattern, the distribution curves of basin storage capacity area and infiltration capacity are assembled vertically, and using Horton formula to calculate Infiltration capacity. The stored-full runoff and superinfiltrating runoff are assembled vertically, which can make the runoff producing model be generality, and can be used in wet areas, arid areas and semi-arid and semi-humid areas.

\subsection{Overland flow simulation model}

Lagging calculus model is used for the calculation of overland sink flow. According to the concept of linear hydrological systems, the role of transfer and regulation of flood wave in the river can be as $\mathrm{n}$ series "linear channel" respectively and $\mathrm{n}$ series "linear reservoir". The former can be only promotion without regulation and storage, and the last can attenuate flood waves. Lagging calculus model can be used for the calculation of the small basin's confluence and the urban's drainage division confluence.

\subsection{River flow concentration model}

According to the river data and the computing accuracy requirements for urban and non-urban, one-dimensional hydrodynamic model is used in the urban area and the Mashingen model is used in the hilly and non-urban to calculate river confluence.

\subsection{Dam and gate operation model}

The gat and dams operation is divided into two types: large and medium-sized reservoir operation and urban gate operation. Based on water balance equation, the operation calculation can use the relationship lines of water level $\sim$ storage volume and water level $\sim$ discharge amount for large and medium - sized reservoirs. The operation method includes plan operation, simulation operation (control and discharge operation, and gate operation) and target operation. Urban gate and dam operation use the weir gate formula, and calculation is done according to the upstream and downstream water level and gate opening. The operation methods include plan operation, given the gate opening operation and control and discharge operation.

\subsection{Waterlogging warning}

By the analysis of Historical Rainstorm and Flood Disaster Data in Beijing, the warning indicators and thresholds of easy dropsy points in city are constructed. Using real-time and forecasting rainfall data and hydrological forecast data, the results of waterlogging warning is given though comprehensive judgment.

\subsection{Model coupling}

The key basin flood control operation system in Beijng relates to the runoff generation and concentration model, river network evolution model, operation analysis model etc.. Especially the coupling between runoff generation and concentration hydrological model and one-dimensional hydrodynamic river network model is the key. This is mainly from the time and space for these models to be coupled.

In the space, by refining the urban drainage / catchment area, the runoff and overland sink flow nodes are coupled with the one - dimensional hydrodynamic section of the river. 
In time, the calculate period for hydrological model generally is hours, but the calculate period for hydrodynamic model is seconds or minutes. The linear interpolation is performed when the results of the hydrological model are docked with the hydrodynamic model, and the hydrodynamic model considers the whole hour data when docking with the hydrological model or the operation model.

\subsection{Model validation}

Using these models to simulate the typical flood process in Beijing in recent years, and the parameters of the model are adjusted to verify the reliability of the model. The simulation results of the "7.20" rainstorm in 2016 and the "7.21" rainstorm in 2012 are shown in Error! Reference source not found. and Error! Reference source not found.. It can be seen from the results that the integrated model can meet the needs of flood control management.
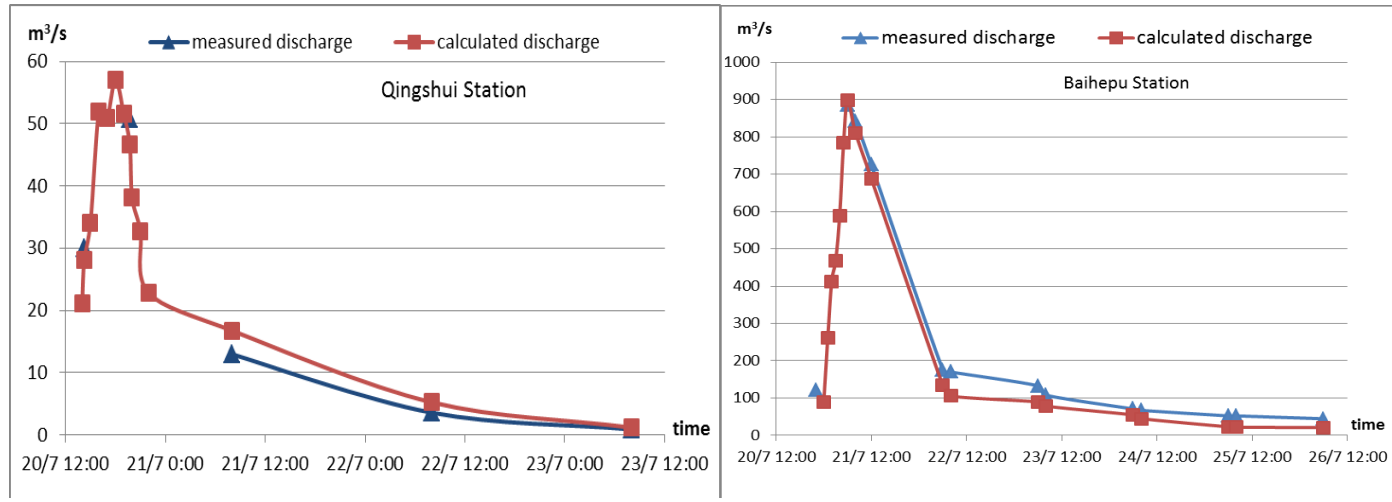

Figure 2: Results of model validation ("7.20" rainstorm in 2016)

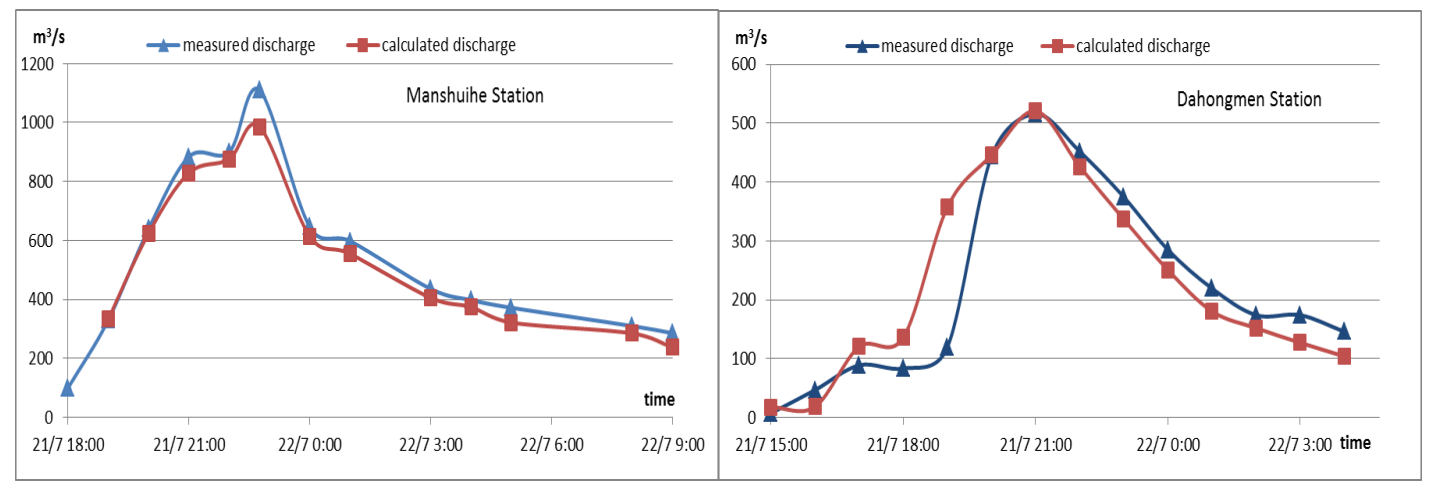

Figure 3: Results of model validation ("7.21" rainstorm in 2012) 


\section{System design and develop}

On the basis of data collection and model building, the system design and development are done. The specific content includes the overall structure, development of technology and the main function.

\subsection{Overall framework of the system}

The key basin flood control operation system in Beijng concludes that user layer, application layer, data layer, infrastructure layer. The architecture is shown by Error! Reference source not found..

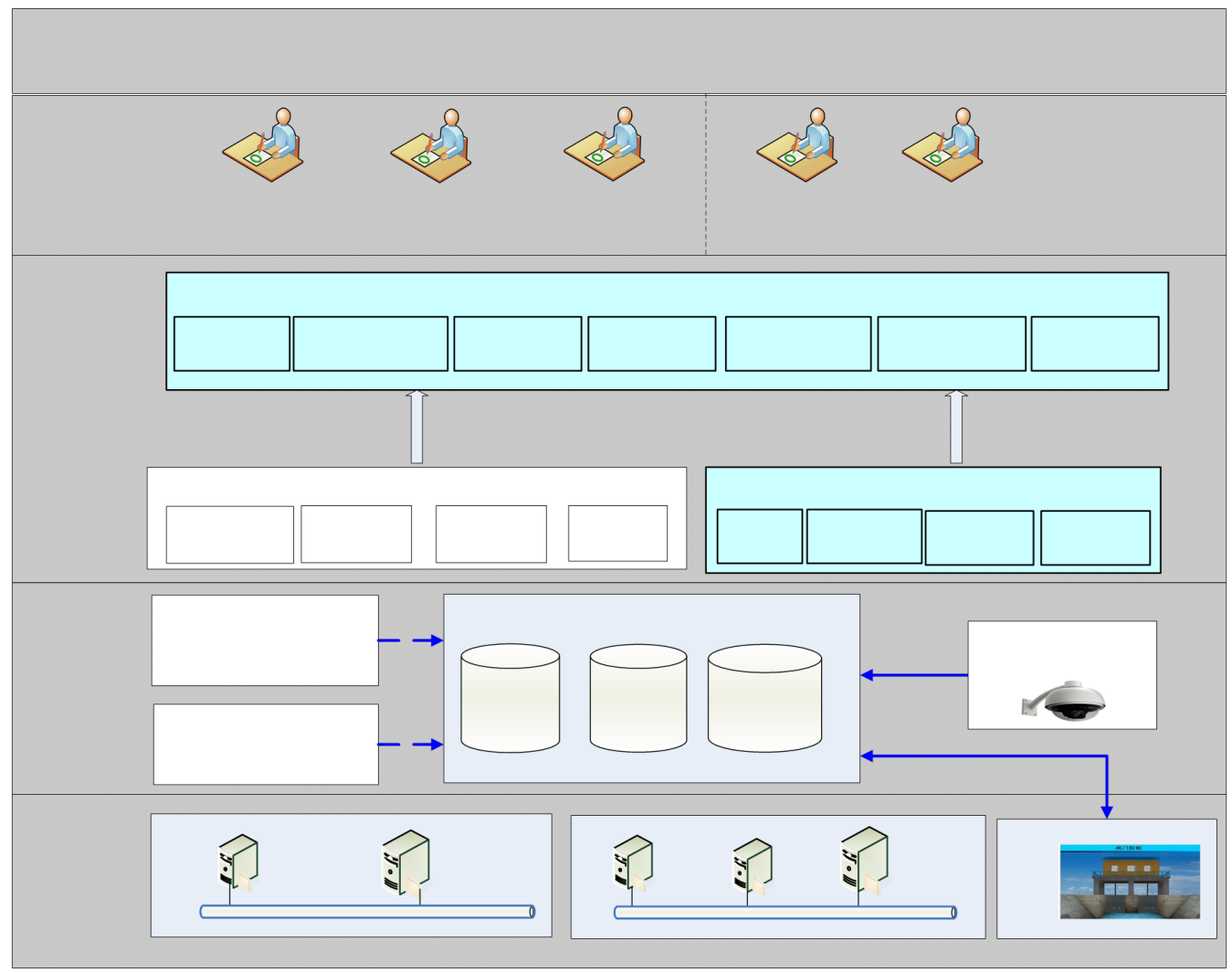

Figure 4: Overall framework of the system

\subsection{Developing technology}

The key basin flood control operation system in Beijng is built by MVC application mode. By this technology architecture, business logic, data Interface can be separated, and the business logic is aggregated into a component. While improving and personalizing interfaces and user interaction at the same time, there is no need to rewrite business logic, which can help for the maintenance and expansion of the system. The technology Architecture of key basin flood control operation system in Beijng concludes the control layer, business logic layer, persistence layer, database. The technical roadmap is shown by Figure 5 . 
Research and Development of Flood Control Operation System of Beijing City, China F. Chai et al.

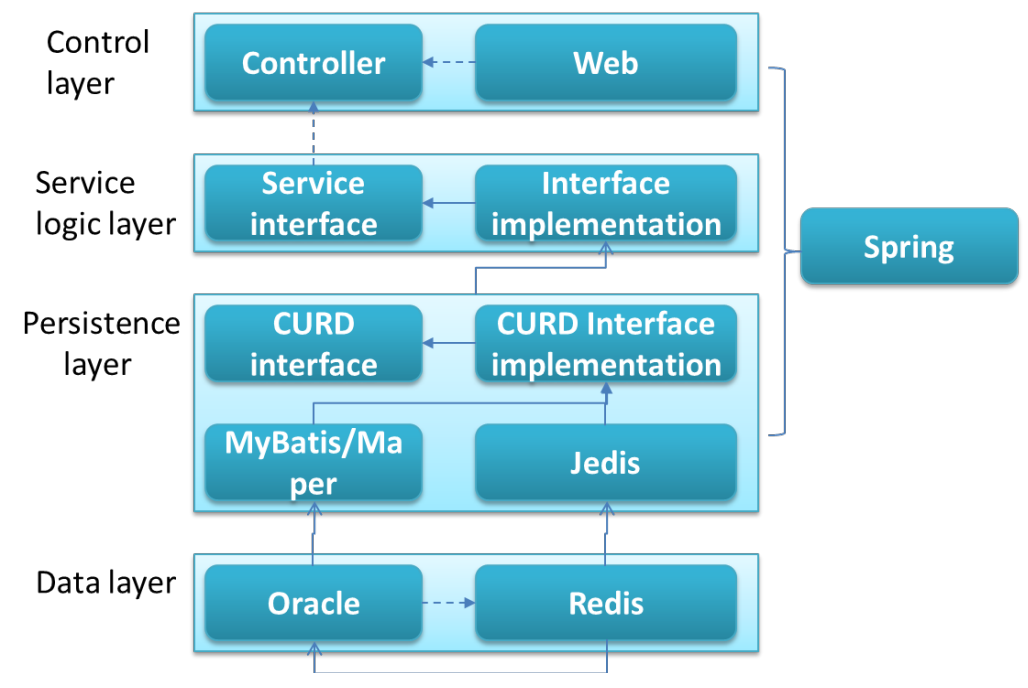

Figure 5: System framework of developing technology

\subsection{Main system function}

The main functions of the system include flood control situation analysis, flood control operation, result evaluation, scheme management, dam and gate monitoring, mobile applications and systems management.

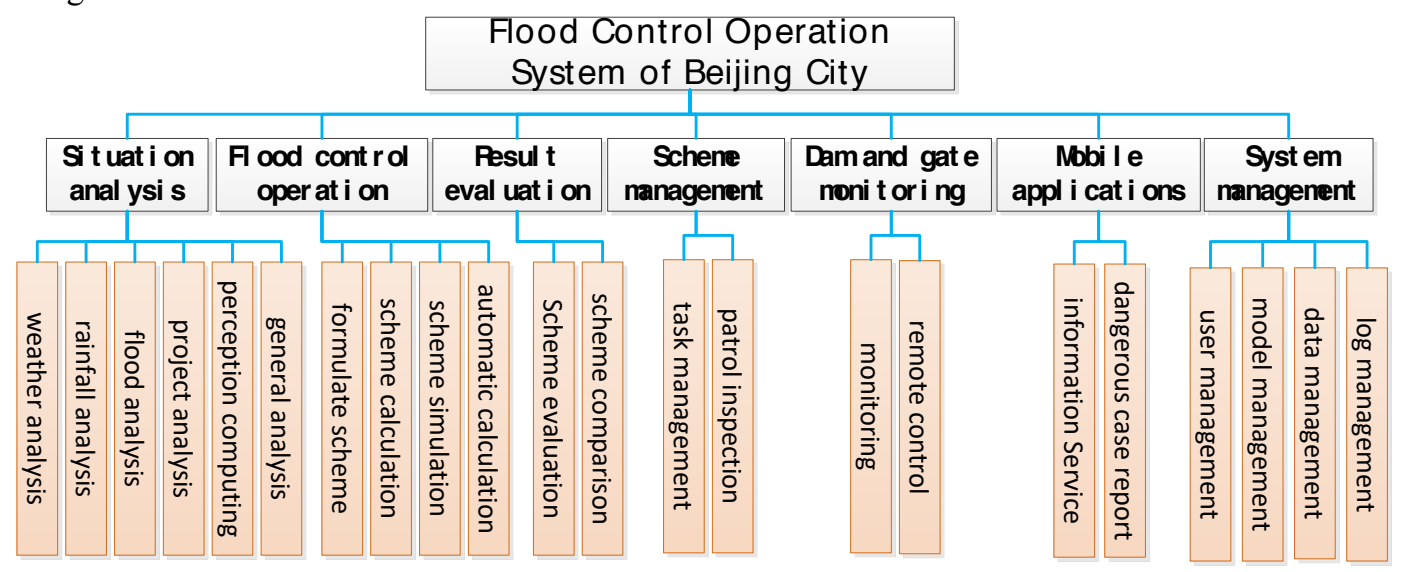

Figure 6: Main function of the system

\subsubsection{Situation analysis}

In the situation analysis function, monitoring and forecasting information of weather, rainfall, water regime etc. are shown, and the system can help us understand and predict the dynamic changes in the flood control situation, and give the comprehensive analysis report automatically. This function was shown in Figure 7. 


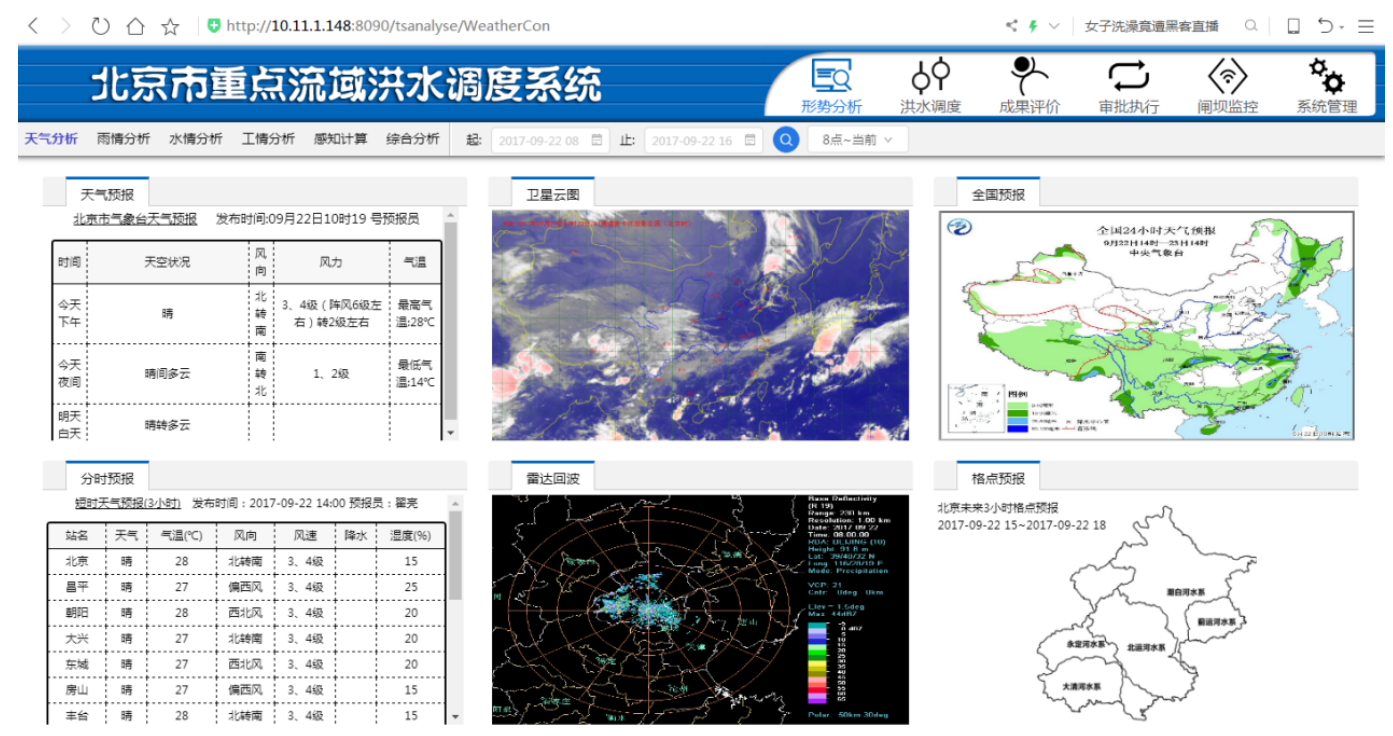

\subsubsection{Flood control operation}

Figure 7: Page of situation analysis

The combined dispatching and whole process simulation of river, reservoir and gate were realized. We can simulate rainfall-runoff, surface convergence, river convergence and reservoir or gate operation by setting or selecting future rainfall, model parameters and scheduling methods of projects. This function was shown in Figure 8.

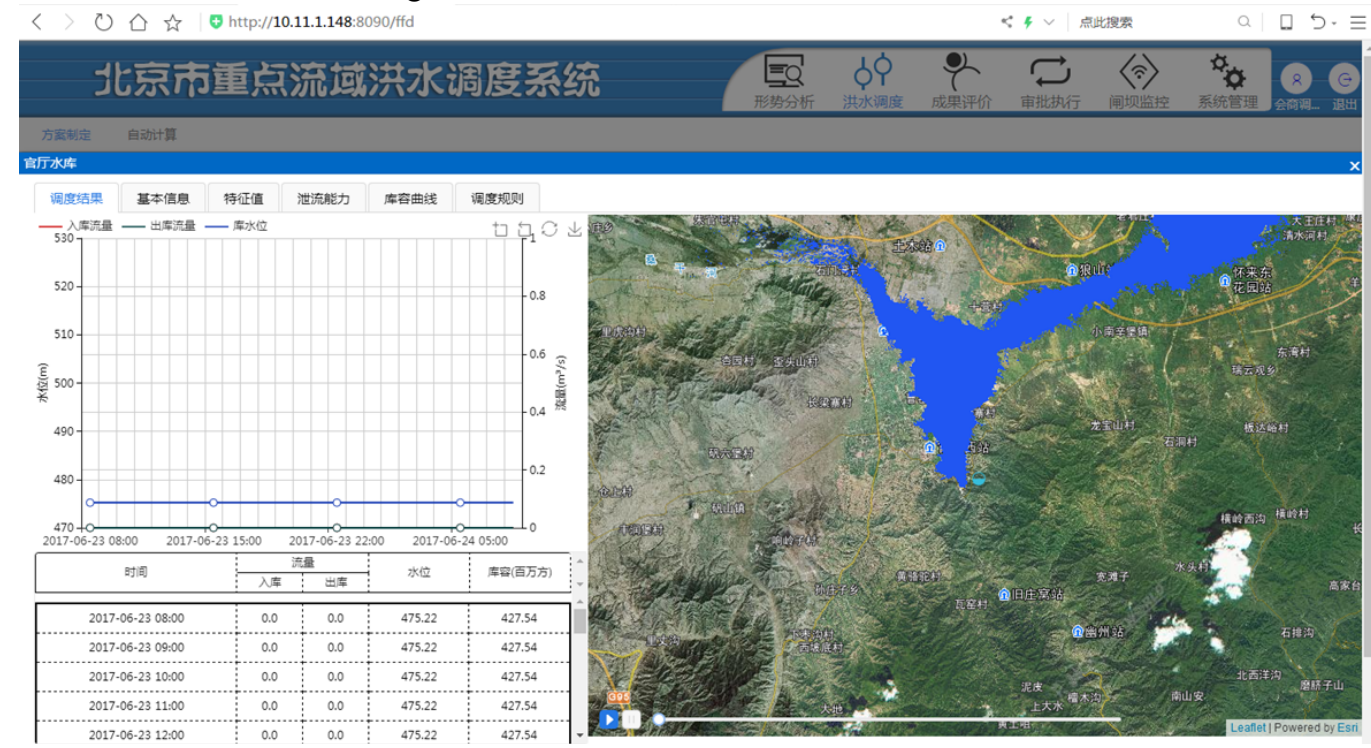

Figure 8: Page of flood control operation

\subsubsection{Result evaluation}

By selecting and setting the evaluation index and weight, the different scheduling results are evaluated, and the recommendations are given for decision makers. And we can manage search, delete or modify the scheduling results. 
Research and Development of Flood Control Operation System of Beijing City, China F. Chai et al.

\subsubsection{Other functions}

In addition to the above functions, it also includes the functions, such as task management, patrol inspection, dam and gate monitoring and remote control, mobile applications and System management.

\section{Conclusions}

Based on the existing hardware and software environment in Beijing, through the integration of various data, the flood forecast and operation model is constructed, and the software system is developed. These aspects that coupling of hydrological hydrodynamic model, external flood operation, waterlogging warning and software design and development all have made innovative achievements. The model and system were deployed in Beijing in June 2017. After the application in the flood season, it ran smoothly, and the calculation accuracy met the flood control requirements, which can support flood control decision work in Beijing.

\section{References}

[1] Guo Sheng-lian, Peng Hui, Wang Jin-xing, et al.. Design and development of reservoir operation system J., Journal of China Hydrology, 2001,(03) , pp.4-7.

[2] Cheng Chun-tian, Ou Chun-ping, Wang Ben-de. Integrated management technology for flood control system of reservoirs J. EI, Journal of Hydraulic Engineering, 2001,(03), pp.77-81.

[3] Sajjad Ahmad, Slobodan P. Simonovic et.al., An Intelligent Decision Support System for Management of Floods, J. Sci, Water Resources Management, 2006, Vol.20 (3), pp.391-410.

[4] Wang Guo-li, Peng Yong, He Bin, et al. Study of B/S \& C/S-based flood control decision-making support system and its application J. EI,. Journal of Dalian University of Technology, 2010,50(02), pp.258-263.

[5] Hu Siyi,Song De-dun, Wu Yong-xiang et al. Overall design of Yangtze River flood control decision support system J. EI,. Advances in Water Science,1996,(04), pp.4-15.

\section{Acknowledgements}

This research was funded by the National Key Research and Development Plan of China (2016YFC0803107). 